\title{
Qualidade de Vida Familiar, Satisfação com a Vida e Apoio Social Percebido na Deficiência Visual
}

\author{
Inês Grilo ${ }^{1}$ \\ CEIFAC-Centro Integrado de Apoio Familiar de Coimbra, Coimbra, Portugal \\ Sofia Major \\ Faculdade de Psicologia e de Ciências da Educação da Universidade de Coimbra, \\ Coimbra, Portugal
}

\begin{abstract}
Resumo
O estudo da influência da deficiência visual no indivíduo permanece ainda com questões por responder. Esta pesquisa comparou a qualidade de vida familiar, satisfação com a vida e apoio social percebido numa amostra de sujeitos adultos com deficiência visual $(n=52)$ e normovisuais $(n=52)$. Verificaram-se diferenças estatisticamente significativas, com o grupo com deficiência visual a apresentar resultados superiores nos fatores Tempo e Emprego do QOL e resultados inferiores no fator Mass Media do QOL e na subescala Vinculação da EPS. Obtiveram-se diferenças estatisticamente significativas entre as variáveis demográficas e a qualidade de vida familiar e o apoio social percebido, nas variáveis sexo (resultado superior no sexo masculino), idade (resultados mais elevados entre 18-35 anos) e situação profissional (resultados superiores em sujeitos empregados), no grupo com deficiência visual. Apesar do contributo deste trabalho, são necessários mais estudos para clarificar a influência da deficiência visual no indivíduo, como também na família.
\end{abstract}

Palavras-chave: Deficiência visual, qualidade de vida familiar, satisfação com a vida, apoio social percebido.

\section{Family Quality of Life, Satisfaction with Life and Perceived Social Support in Visual Impairment}

\begin{abstract}
The study of the influence of visual impairment on the individual still remains with unanswered questions. This research compared the quality of family life, life satisfaction and perceived social support in a sample of adult with visual impairment $(n=52)$ and sighted $(n=52)$ using. There were obtained statistically significant differences with the group with visual impairment providing superior results in the factors Time and Employment of the QOL, lower results in the factor Mass Media of the QOL and the subscale Attachment of the EPS. There was obtained statistically significant differences between de-

Endereço para correspondência: Rua da Guiné, n. ${ }^{\text {39 }}$, 1. ${ }^{\circ}$ Esq., 3030-054 Coimbra, Portugal. E-mail: imsgrilo@ gmail.com e smajor@fpce.uc.pt

O presente artigo foi escrito com base na Dissertação de Mestrado Integrado em Psicologia, na área de especialização em Psicologia Clínica e da Saúde, subárea de especialização em Sistémica, Saúde e Família da primeira autora com orientação da segunda, apresentada na Faculdade de Psicologia e de Ciências da Educação da Universidade de Coimbra.

Agradecimento: As autoras do artigo gostariam de expressar o seu agradecimento para com a Associação dos Cegos e Amblíopes de Portugal (ACAPO), em especial, ao Dr. José Mário Albino e à ACAPO - Coimbra, que recebeu prontamente este projeto, e a todos os participantes com deficiência visual e normovisuais pelo seu precioso contributo para este estudo.
\end{abstract}


mographic variables and the quality of family life and perceived social support, on the variables gender (higher result in males), age (higher results from 18-35 years), and employment status (superior results in employed subjects), in the group with visual disabilities. Despite the contribution of this study, further studies are needed to clarify the influence of visual impairment on the individual but also the family.

Keywords: Visual impairment, family quality of life, satisfaction with life, perceived social support.

\section{Calidad de la Vida Familiar, Satisfacción con la Vida y el Apoyo Social Percibido en la Discapacidad Visual}

\section{Resumen}

El estudio de la influencia de la discapacidad visual en el individuo permanece aún con preguntas sin respuesta. Este estudio comparó la calidad de la vida familiar, satisfacción con la vida y el apoyo social percibido en una muestra de sujetos adultos con discapacidad visual $(n=52)$ y normovisuais $(n=52)$. Se comprobaron diferencias estadísticamente significativas, con el grupo con deficiencia visual mostrando resultados superiores en los factores Tiempo y Empleo del QOL, y menores resultados en los Mass Media del QOL y la subescala de Vinculación de la EPS. Se han encontrado diferencias estadísticamente significativas entre las variables demográficas y la calidad de la vida familiar y el apoyo social percibido, en las variables sexo (mayor resultado en los hombres), edad (resultados mayores de 18-35 años) y situación laboral (resultados superiores en los sujetos empleados), en el grupo con discapacidades visuales. A pesar de la contribución de este estudio, son necesarios más estudios para aclarar la influencia de la discapacidad visual en el individuo, sino también en la familia.

Palabras clave: Discapacidad visual, la calidad de vida familiar, la satisfacción con la vida, apoyo

social percibido.

Nas últimas três décadas o estudo da deficiência visual tem contribuído para a compreensão do seu caráter crónico e do seu impacto, não apenas a nível individual, como também familiar, comunitário e no próprio sistema de saúde (Bambara et al., 2009). Neste sentido, a Organização Mundial de Saúde (World Health Organization [WHO], 2012) considera que a deficiência visual causa limitações ao nível da execução de tarefas quotidianas, com consequências negativas para a qualidade de vida destes indivíduos e suas famílias. Segundo a Classificação Estatística Internacional das Doenças e Problemas Relacionados com a Saúde - 10 Revisão (WHO, 2004) existem três níveis de função visual: a visão normal, a baixa visão (deficiência visual moderada e grave) e a cegueira. Assim, a baixa visão se caracteriza pela incapacidade visual que, com ou sem o auxílio de aparelhos tecnológicos (e.g., lupa, ecrã aumentado), permite ao indivíduo realizar algumas tarefas para as quais a visão é fundamental, não se tornando funcional através de intervenção médica (e.g., cirúrgica) especializada, enquanto a cegueira é a incapacidade de ver (WHO, 2013).

De acordo com o paradigma sistémico da doença crónica de Rolland (1994), a cegueira aguda, especificamente estudada por este autor, se caracteriza por: (a) ter começo agudo, já que as mudanças afetivas e instrumentais por que passam indivíduos e famílias ocorrem dentro de um curto período de tempo; (b) ser de curso constante, dando lugar a uma estabilização da evolução biológica da doença após o momento inicial em que esta ocorre, adquirindo uma limitação funcional mais ou menos acentuada; (c) não ser fatal; e (d) ser incapacitante ao nível sensorial com a consequência mais óbvia da perda de visão total ou parcial nos seus diferentes graus, podendo estar inerente uma grande ativação emocional (e.g., choque, desespero, tristeza, irritabilidade, medo), em parte, devido ao estigma de ser "cego" (Silveira \& Sequeira, 2002). É dentro destes indicadores que se torna pertinente atualizar o entendimento sobre a deficiência 
visual - ou seja, baixa visão e cegueira - bem como acerca dos tipos de apoio disponíveis para esta população (e.g., instrumental, financeiro, deslocações) e quem os pode prestar (e.g., família, instituições). O presente estudo procurou compreender em que medida a deficiência visual poderá influenciar os indivíduos ao nível da qualidade de vida familiar, satisfação com a vida e apoio social percebido.

$\mathrm{O}$ estudo da qualidade de vida familiar permite avaliar a percepção e a interação da família como um todo (Hu, Summers, Turnbull, \& Zuna, 2011) através de componentes emocionais, cognitivas e sociais que permitem a construção e manutenção de redes de apoio social, podendo ainda estar positivamente correlacionada com o sucesso do processo de reabilitação de pessoas com deficiência visual (Stelmack, 2001). Por seu lado, a avaliação da qualidade de vida possibilita verificar a percepção que o indivíduo possui da medida em que determinadas condições (e.g., socioeconómicas, culturais, saúde) influenciam a sua vida (Bittencourt \& Hoehne, 2006; Fagulha, Duarte, \& Miranda, 2000). Com efeito, várias foram as pesquisas que procuraram avaliar o impacto da deficiência visual na qualidade de vida individual, todavia, denota-se uma evidente escassez de pesquisas focadas na questão da qualidade de vida familiar. Na pesquisa realizada por Bittencourt e Hoehne (2006), os resultados indicaram a percepção positiva da qualidade de vida por parte de sujeitos adultos com deficiência visual, considerando que a elevada escolaridade da maioria da amostra estudada possa ter tido um papel preponderante nestes resultados, embora não tenham sido feitos estudos para averiguar a existências de diferenças estatisticamente significativas. Por outro lado, os resultados obtidos no estudo de Langelaan e colaboradores (2007) destacaram um impacto tendencialmente negativo da deficiência visual na qualidade de vida dos sujeitos, em parte devido a uma maior percepção das dificuldades associadas à ausência parcial ou total de acuidade visual no quotidiano. No que diz respeito à existência de diferenças estatisticamente significativas na qualidade de vida de adultos com baixa-visão atendendo a determinadas variáveis demográficas, tais como o sexo, a pesquisa elaborada por Holbrook, Caputo, Perry, Fuller e Morgan (2009) indica uma tendência para as mulheres obterem resultados relativamente mais baixos do que os homens.

Fagulha et al. (2000) consideram existir uma relação teórica entre o conceito de qualidade de vida e a satisfação com a mesma, procurando contextualizar a investigação realizada sobre a satisfação com a vida em sujeitos com deficiência visual. Neste sentido, o conceito de satisfação com a vida veio contribuir para a definição de outros conceitos como o de bem-estar subjetivo (A. Simões, 1992) ou de qualidade de vida (Fagulha et al., 2000). A satisfação com a vida representa, portanto, um fator cognitivo do bem-estar subjetivo (Fagulha et al., 2000; A. Simões, 1992), fundamental pela possibilidade de precaver eventuais distúrbios de caráter físico ou psicológico que possam estar associados a comportamentos de risco para com a própria vida (Fagulha et al., 2000). Assim, da pesquisa realizada sobre a avaliação da satisfação com a vida em sujeitos adultos portadores de deficiência visual pode destacar-se o estudo realizado por Cimarolli e Wang (2006) que obteve uma maior satisfação com a vida por parte de sujeitos com deficiência visual empregados do que sujeitos desempregados. Também o estudo de Good, La Grow e Alpass (2008) indicou a existência de uma diferença estatisticamente significativa relativamente à satisfação com a vida, com sujeitos com deficiência visual a obterem resultados tanto mais elevados quanto maior a idade, contrariamente à amostra normovisual, o que, segundo os autores, pode ser explicado através da capacidade de resiliência e flexibilidade acumuladas ao longo dos anos pelos sujeitos com deficiência visual, representando assim uma estratégia de coping (Good et al., 2008). No mesmo sentido, o estudo de Guerette e Smedema (2011) verificou que os sujeitos com deficiência visual mais velhos em situação de empregabilidade obtinham resultados mais elevados na percepção da satisfação com a vida.

Quanto ao apoio social percebido, este abrange um conjunto de recursos alcançados em contexto de relações sociais, a que os sujeitos podem aceder em situações geradoras de stress 
(Moreira \& Canaipa, 2007). Neste contexto, o estudo efetuado por La Grow, Alpass, Stephens e Towers (2011) pretendeu analisar a relação entre qualidade de vida e outras dimensões tais como as provisões sociais e satisfação com a vida, numa amostra de idosos com deficiência visual. Os resultados elevados ao nível da satisfação com a vida se encontravam associados a resultados superiores na percepção da qualidade de vida, mas não com o apoio social percebido, possivelmente devido ao fato de as pessoas com deficiência visual associarem o apoio prestado por outros à dependência e à falta de autonomia em vários níveis (e.g., económico, mobilidade, isolamento social; La Grow et al., 2011).

O presente estudo tem como objetivo geral a comparação da qualidade de vida familiar, da satisfação com a vida e do apoio social percebido numa amostra de sujeitos adultos com deficiência visual e noutra de normovisuais. Pretende-se, especificamente, analisar: (a) a consistência interna dos itens dos instrumentos de avaliação utilizados numa amostra de sujeitos adultos com deficiência visual; (b) a existência de diferenças estatisticamente significativas relativas à percepção da qualidade de vida familiar, satisfação com a vida e apoio social percebido entre os dois grupos (deficiência visual e normovisão); e (c) a influência de variáveis demográficas (e.g., sexo, idade e situação profissional) nos resultados obtidos no grupo de adultos com deficiência visual.

\section{Método}

\section{Amostra}

Para a realização deste estudo foram necessárias duas amostras de sujeitos com idade igual ou superior a 18 anos: sujeitos adultos portadores de diagnóstico de deficiência visual moderada a total (grupo DV) e sujeitos adultos normovisuais (grupo de comparação). A amostra do grupo DV se constituiu por 52 sujeitos igualmente distribuídos pela variável sexo $(50 \%$ feminino e $50 \%$ masculino), com uma média de 45,38 anos $(D P=13,80)$, dos quais $78,8 \%$ cegos, e 21,2\% com baixa visão. Quanto à situação profissional, $28,8 \%$ eram aposentados e $44,2 \%$ empregados, de destacar que $34,6 \%$ dos sujeitos concluiu o Ensino Superior. A amostra de comparação se compôs por 52 sujeitos normovisuais, com uma média de 43,10 anos $(D P=12,12)$ igualmente distribuídos pela variável sexo. Destes sujeitos, 44,2\% concluiu o Ensino Superior, $84,6 \%$ se encontrava empregado e apenas $1,9 \%$ aposentado. Analisada a equivalência entre os dois grupos quanto a variáveis como idade, escolaridade e situação profissional, não foram verificadas diferenças estatisticamente significativas para as variáveis idade, $\chi^{2}(5, N=104)=7,36, p<$ 0,195 , e escolaridade, $\chi^{2}(2, N=104)=1,302, p<$ 0,522 . A variável situação profissional se revelou estatisticamente significativa, $\chi^{2}(2, N=104)$ $=20,466, p<0,001$, no sentido de o grupo DV apresentar mais sujeitos em situação de reforma do que o grupo de comparação, e menos sujeitos em situação de empregabilidade.

Quanto às variáveis de apoio recebido pelos sujeitos do grupo DV, $75 \%$ já recebeu apoio institucional, sendo a reabilitação $(48,1 \%)$ e o apoio material $(23,1 \%)$ os tipos de apoio mais mencionados. $\mathrm{O}$ apoio informal dos progenitores é o mais referido $(34,6 \%)$.

\section{Procedimento de Coleta e Considerações Éticas}

Para a recolha da amostra do grupo DV, foi solicitada colaboração à Delegação de Coimbra da Associação dos Cegos e Amblíopes de Portugal (ACAPO), tendo o seu representante máximo autorizado a presente pesquisa. Realizou-se uma divulgação da pesquisa a nível nacional através das várias delegações da ACAPO, ainda que alguns sujeitos tenham sido contactados a partir das suas redes interpessoais. A amostra do grupo de comparação foi recolhida através de uma amostra de conveniência, atendendo a sujeitos com características demográficas próximas (e.g., sexo, idade, e nível de escolaridade) dos sujeitos do grupo DV. Após a identificação de possíveis sujeitos interessados em participar no estudo, em ambas as amostras, se procedeu ao contacto presencial ou telefónico com os mesmos, num horário previamente agendado atendendo à sua disponibilidade, lhes tendo sido explicado e lido o documento de consentimento informado que apresentava os 
objetivos da presente investigação. Assim, o protocolo foi aplicado aos participantes numa mesma ordem pré-definida com o intuito de assegurar a estandardização dos procedimentos. No que se refere à amostra de pessoas com deficiência visual, a aplicação do protocolo foi efetuada por contacto telefónico a $90,4 \%$ dos sujeitos, com uma duração aproximada de 25 minutos.

\section{Instrumentos}

Questionário Demográfico. Foram construídas duas versões deste questionário, cada uma adaptada às amostras recolhidas, com uma parte comum de dados pessoais dos sujeitos (e.g., idade, estado civil, localidade de residência, escolaridade e composição do agregado familiar). A versão desenvolvida para o grupo DV incluía, ainda, dois grupos específicos de questões: apoio recebido por parte de instituições/familiares (e.g., tipo de apoio institucional recebido, tipo de apoio informal recebido, frequência do apoio), e informações acerca do diagnóstico de DV.

Qualidade de Vida (QOL). A qualidade de vida familiar foi avaliada com recurso à versão portuguesa do Quality of Life, construído por Olson e Barnes em 1982, Qualidade de Vida (QOL; Versão Núcleo de Seguimento Infantil e Acção Familiar [Nusiaf-sistémica], adaptado, 2007; validado J. M. L. Simões, 2008). O QOL é constituído por 40 itens, repartidos por 11 fatores (e.g., Bem-Estar Financeiro, Relações Sociais e Saúde, Família e Conjugalidade), abrangidos por uma mesma questão ("Qual o seu grau de satisfação com?", por exemplo, "A sua familia"), sendo que cada item é cotado segundo uma escala de Likert com 5 níveis (1 - Insatisfeito; 2 - Pouco satisfeito; 3 - Geralmente satisfeito; 4 - Muito satisfeito; 5 - Extremamente satisfeito). Os estudos de análise fatorial da versão portuguesa do QOL identificaram igualmente $11 \mathrm{fa}-$ tores, que explicam $70,5 \%$ do total de variância, mas apenas 38 itens foram incluídos na composição fatorial. De acordo com a pesquisa elaborada por J. M. L. Simões (2008), os itens 25 e 32 estariam a reduzir o valor da consistência interna dos fatores 7 e 1 , respetivamente, pelo que o resultado do coeficiente alfa de Cronbach desses fatores poderia ser maior caso esses dois itens fossem excluídos. Já os estudos de consistência interna da versão portuguesa do QOL apontaram para um valor de coeficiente alfa de Cronbach de 0,92 para a escala total e entre 0,74 e 0,98 para os vários fatores (J. M. L. Simões, 2008).

Escala de Satisfação com a Vida (SWLS). A satisfação com a vida foi avaliada através da versão portuguesa da Satisfaction with Life Scale (SWLS), construída por Diener, Emmons, Larsen e Griffin, em 1985, e validada para português por A. Simões (1992) - versão utilizada neste estudo -, composta por cinco itens, cotados numa escala de Likert com cinco níveis de resposta $(1$ - Discordo muito a 5 Concordo muito). A pontuação total obtida na SWLS oscila entre 5 e 25 , sendo que quanto mais elevado for o resultado, maior será a satisfação com a vida. No estudo da consistência interna foi obtido um valor satisfatório, com um coeficiente alfa de Cronbach de 0,77 (A. Simões, 1992). Por sua vez, na validação da versão portuguesa procedeu-se ao estudo do grau de associação entre a SWLS, a satisfação diária e felicidade, do Subjective Well-Being (SWB), se verificando que todas as correlações foram positivas $(p<$ 0,01), apresentando bons índices de validade preditiva (A. Simões, 1992).

Escala de Provisões Sociais (EPS). O Apoio Social Percebido foi avaliado através da Escala de Provisões Sociais, adaptada por Moreira e Canaipa (2007) da versão original Social Provisions Scale, construída por Cutrona e Russel, em 1987. A EPS é uma escala de avaliação de autorresposta composta por 24 itens distribuídos equitativamente por dois fatores (Apoio Íntimo e Apoio Casual), organizados por um total de seis subescalas (e.g., Aconselhamento, Vinculação, Oportunidade de Prestação de Cuidados), em que cada item é cotado de acordo com uma escala de Likert com quatro níveis de resposta (1 - Discordo fortemente a 4 - Concordo fortemente), com dois itens invertidos por fator. No estudo da consistência interna se alcançou um valor de coeficiente alfa de Cronbach de 0,91 (Moreira \& Canaipa, 2007). Relativamente à validação da versão portuguesa, foi realizado o estudo do grau de associação entre a EPS e o Questionário de Apoio Social (QASR) se verificando correla- 
ções positivas, ao passo que no estudo do grau de associação entre a EPS e a Escala de Desejabilidade Social de Marlowe-Crowne (EMC) não foi obtida qualquer correlação significativa, permitindo concluir que a validade convergente e discriminante apresentada pela EPS a torna numa boa medida para a avaliação das diversas subescalas do apoio social percebido (Moreira \& Canaipa, 2007).

\section{Procedimentos Estatísticos}

As análises descritivas e inferenciais foram efetuadas com recurso ao software IBM SPSS (Statistical Package for the Social Sciences, Version 20.0 for Windows). A análise descritiva das amostras foi realizada através do estudo das frequências e percentagens. A equivalência entre o grupo DV e o grupo de comparação foi avaliada pelo teste de ajustamento do Qui-Quadrado. Foi utilizado o coeficiente alfa de Cronbach para avaliar a consistência interna dos itens do QOL, SWLS e EPS na amostra DV. A existência de diferenças estatisticamente significativas nas médias do grupo DV e do grupo de comparação em termos das três escalas utilizadas foi averiguada pelo teste $t$ de student para amostras independentes. A existência de diferenças nos resultados obtidos no grupo DV, atendendo às variáveis demográficas, foi avaliada através do teste $t$ de student para amostras independentes (e.g., sexo) e da análise de variância (ANOVA, com teste de comparações múltiplas de Bonferroni; e.g., situação profissional). Quando identificadas diferenças estatisticamente significativas, se calculou a magnitude do efeito através da fórmula do eta squared (Pallant, 2005).

\section{Resultados}

\section{Estudo da Consistência Interna}

$\mathrm{O}$ estudo da consistência interna para a totalidade da escala do QOL no grupo DV indicou um valor muito elevado de coeficiente alfa de Cronbach de 0,90, tal como ocorreu no grupo de sujeitos normovisuais $(\alpha=0,91)$. Todavia, os resultados obtidos em ambas as amostras na escala total e nos respetivos fatores são tenden- cialmente inferiores aos resultantes da validação da versão portuguesa do QOL (Tabela 1). Na escala de Satisfação com a Vida (SWLS; A. Simões, 1992) o valor do alfa de Cronbach para a amostra DV foi de 0,83 , e de 0,87 para o grupo de comparação, superior em ambos os grupos ao valor de consistência interna obtido na validação da versão portuguesa $(\alpha=0,77)$. O estudo de consistência interna para a escala total da EPS (Moreira \& Canaipa, 2007) no grupo DV indicou um coeficiente alfa de Cronbach de 0,92, e na amostra de normovisuais de 0,94 . Os resultados obtidos de coeficiente alfa de Cronbach na escala total e respetivas subescalas para o grupo DV são tendencialmente superiores nos dois grupos em comparação com os valores obtidos para a validação da versão portuguesa da EPS, com exceção de três fatores (Tabela 1). Os valores obtidos para o coeficiente alfa de Cronbach remetem assim para um bom funcionamento dos itens dos três instrumentos utilizados no presente estudo.

\section{Qualidade de Vida Familiar, Satisfação com a Vida e Apoio Social Percebido: Deficiência Visual e Normovisão}

Conforme consta da Tabela 2, existem diferenças estatisticamente significativas entre $o$ grupo DV e o grupo de comparação ao nível da qualidade de vida familiar, nos fatores Tempo, $t(102)=3,19, p=0,002$, Mass Media, $t(102)=$ $-2,52, \mathrm{p}=0,013$ e Emprego, $t(102)=1,97, p=$ 0,051 . A diferença surge no sentido de o grupo DV apresentar resultados superiores ao nível da qualidade de vida familiar relacionada com o Tempo $(M=12,98, D P=2,64)$ do que no grupo de comparação $(M=11,13, D P=3,24),\left(\eta^{2}\right.$ $=0,091)$, tal como se verifica no fator Emprego $\left(M_{\text {grupo DV }}=7,12, D P=1,57 ; \mathrm{M}_{\text {grupo comparação }}=6,50\right.$, $D P=1,62),\left(\eta^{2}=0,037\right)$. Pelo contrário, no fator Mass Media o grupo DV obteve uma média inferior $(M=7,23, D P=1,86)$ à do grupo de comparação $(M=8,12, D P=1,71),\left(\eta^{2}=0,059\right)$. Para os restantes fatores referentes à qualidade de vida familiar não foram verificadas diferenças estatisticamente significativas.

Em relação à satisfação com a vida, apesar de o grupo de comparação ter um resultado ten- 
Tabela 1

Estudo Comparativo: Resultados do Coeficiente Alfa de Cronbach QOL e EPS

\begin{tabular}{|c|c|c|c|c|}
\hline Fatores & N. ${ }^{o}$ de itens & $\begin{array}{c}\alpha \\
\text { Grupo DV }\end{array}$ & $\begin{array}{c}\alpha \\
\text { Grupo } \\
\text { Normovisual }\end{array}$ & $\begin{array}{c}\alpha \\
\text { Versão Portuguesa }\end{array}$ \\
\hline QOL & & & & (Simões, 2008) \\
\hline Bem-estar Financeiro & 6 & 0,81 & 0,89 & 0,89 \\
\hline Tempo & 4 & 0,74 & 0,88 & 0,98 \\
\hline Vizinhança e Comunidade & 6 & 0,67 & 0,86 & 0,89 \\
\hline Casa & 5 & 0,81 & 0,80 & 0,90 \\
\hline Mass Media & 3 & 0,58 & 0,52 & 0,81 \\
\hline Relações Sociais e Saúde & 4 & 0,55 & 0,55 & 0,74 \\
\hline Emprego & 2 & 0,62 & 0,72 & 0,74 \\
\hline Religião & 2 & 0,79 & 0,81 & 0,97 \\
\hline Família e Conjugalidade & 2 & 0,52 & 0,57 & 0,80 \\
\hline Filhos & 2 & 0,33 & 0,57 & 0,80 \\
\hline Educação & 2 & 0,78 & 0,66 & 0,83 \\
\hline QOL Escala Total & 40 & 0,90 & 0,91 & 0,92 \\
\hline EPS & & & & $\begin{array}{c}\text { (Moreira \& Canaipa, } \\
\text { 2007) }\end{array}$ \\
\hline Aconselhamento & 4 & 0,75 & 0,78 & 0,71 \\
\hline Aliança Fiável & 4 & 0,82 & 0,83 & 0,76 \\
\hline Reafirmação de Valor & 4 & 0,58 & 0,71 & 0,70 \\
\hline Oportunidade de Prestação de Cuidados & 4 & 0,69 & 0,58 & 0,66 \\
\hline Vinculação & 4 & 0,69 & 0,63 & 0,66 \\
\hline Integração Social & 4 & 0,52 & 0,73 & 0,75 \\
\hline Apoio Íntimo & 16 & 0,91 & 0,92 & 0,88 \\
\hline Apoio Casual & 8 & 0,72 & 0,82 & 0,82 \\
\hline Escala Total & 24 & 0,92 & 0,94 & 0,91 \\
\hline
\end{tabular}

dencialmente superior, a diferença não alcança o limiar de significância estatística. No apoio social percebido, não obstante a tendência do grupo de comparação em ter resultados ligeiramente superiores, somente na subescala Vinculação foi alcançada uma diferença estatisticamente significativa, $t(102)=-2,90, p=0,005$, apresentando o grupo de comparação uma média superior $(M$ $=13,48, D P=1,73)$ ao grupo $\mathrm{DV}(M=12,42$, $D P=1,98),\left(\eta^{2}=0,076\right.$; Tabela 2$)$.

\section{Sexo, Idade e Situação Profissional: Diferenças na Deficiência Visual}

No que diz respeito à variável sexo, se verificou que ao nível da qualidade de vida familiar (avaliada pelo QOL), os homens $(M=14,73, D P$ $=2,29$ ) apresentam resultados significativamente superiores no fator Relações Sociais e de Saúde do que as mulheres $(M=13,23, D P=2,46), t(50)$ $=2,28, p=0,027,\left(\eta^{2}=0,09\right)$. Merece destaque, ainda, o fato de o resultado do fator Mass Media 
Tabela 2

Diferenças na Qualidade de Vida Familiar, Satisfação com a Vida e Apoio Social Percebido: Grupo DV e Grupo Normovisual

\begin{tabular}{|c|c|c|c|c|c|c|}
\hline \multirow{2}{*}{ Resultado } & \multicolumn{2}{|c|}{ Grupo DV } & \multicolumn{2}{|c|}{ Grupo Normovisual } & \multirow{2}{*}{$t$} & \multirow{2}{*}{$p$} \\
\hline & $M$ & $D P$ & $M$ & $D P$ & & \\
\hline \multicolumn{7}{|l|}{ QOL } \\
\hline Bem-estar Financeiro & 16,90 & 4,00 & 16,31 & 4,97 & 0,68 & 0,502 \\
\hline Tempo & 12,98 & 2,64 & 11,13 & 3,24 & 3,19 & 0,002 \\
\hline Vizinhança e Comunidade & 18,21 & 3,20 & 17,92 & 3,97 & 0,41 & 0,684 \\
\hline Casa & 18,83 & 3,51 & 18,33 & 3,11 & 0,77 & 0,444 \\
\hline Mass Media & 7,23 & 1,86 & 8,12 & 1,71 & $-2,52$ & 0,013 \\
\hline Relações Sociais e Saúde & 13,98 & 2,47 & 14,06 & 2,25 & $-0,17$ & 0,869 \\
\hline Emprego & 7,12 & 1,57 & 6,50 & 1,62 & 1,97 & 0,051 \\
\hline Religião & 6,75 & 1,53 & 6,35 & 1,45 & 1,38 & 0,171 \\
\hline Família e Conjugalidade & 7,94 & 1,56 & 7,98 & 1,46 & $-0,13$ & 0,897 \\
\hline Filhos & 7,33 & 1,64 & 7,15 & 2,10 & 0,47 & 0,641 \\
\hline Educação & 6,60 & 1,54 & 6,58 & 2,01 & 0,06 & 0,956 \\
\hline Total QOL & 130,75 & 16,65 & 126,85 & 17,30 & 1,17 & 0,244 \\
\hline \multicolumn{7}{|l|}{ SWLS } \\
\hline Total ESV & 16,23 & 4,24 & 17,79 & 4,58 & $-1,80$ & 0,075 \\
\hline \multicolumn{7}{|l|}{ EPS } \\
\hline Aconselhamento & 13,10 & 1,95 & 13,60 & 1,87 & $-1,33$ & 0,185 \\
\hline Aliança Fiável & 13,33 & 1,87 & 13,90 & 1,92 & $-1,55$ & 0,124 \\
\hline Reafirmação Valor & 12,42 & 1,51 & 12,63 & 1,76 & $-0,66$ & 0,513 \\
\hline Oportunidade Prestação Cuidados & 13,25 & 1,70 & 13,58 & 1,61 & $-1,01$ & 0,317 \\
\hline Vinculação & 12,42 & 1,98 & 13,48 & 1,73 & $-2,90$ & 0,005 \\
\hline Integração Social & 12,71 & 1,38 & 13,13 & 1,80 & $-1,34$ & 0,182 \\
\hline Apoio Íntimo & 52,10 & 6,46 & 54,56 & 6,46 & $-1,94$ & 0,055 \\
\hline Apoio Casual & 25,13 & 2,58 & 25,77 & 3,22 & $-1,11$ & 0,270 \\
\hline Total EPS & 77,23 & 8,64 & 80,33 & 9,22 & $-1,77$ & 0,080 \\
\hline
\end{tabular}

ser idêntico para os dois sexos. Relativamente às dimensões satisfação com a vida e apoio social percebido não foram obtidas diferenças estatisticamente significativas para esta variável.

Para facilitar a análise e interpretação dos resultados da variável idade, promovendo uma distribuição equitativa e equilibrada do número de sujeitos da amostra DV pelas diferentes categorias etárias, foram definidas três classes: 18-
35 anos, 36-50 anos, e 51 ou mais anos. Nesta variável se identificaram diferenças estatisticamente significativas apenas ao nível do apoio social percebido (avaliado pela EPS), $\left(\eta^{2}=0,13\right.$ a 0,27), nomeadamente nas subescalas Aconselhamento, $F(2,49)=5,66, p=0,006$; Aliança Fiável, $F(2,49)=7,80, p=0,001$; Oportunidade de Prestação de Cuidados, $F(2,49)=7,92, p=$ 0,001 ; Vinculação, $F(2,49)=5,54, p=0,007$; In- 
tegração Social, $F(2,49)=5,76, p=0,006$; Apoio Íntimo, $F(2,49)=9,07, p<0,001$; Apoio Casual, $F(2,49)=3,58, p=0,035$; e Escala Total da EPS, $F(2,49)=7,92, p=0,001$. O teste de Bonferroni revelou um resultado mais elevado nas subescalas Aconselhamento, Aliança Fiável, Oportunidade de Prestação de Cuidados, Apoio Íntimo e na Escala Total da EPS por parte de sujeitos DV com idades compreendidas entre os 18 e os 50 anos do que os sujeitos DV com mais de 51 anos. Por sua vez, os sujeitos DV dos 18 aos 35 anos obtiveram resultados superiores nas subescalas Vinculação, Integração Social e Apoio Casual do que os sujeitos DV com mais de 51 anos.

Quanto à variável situação profissional, foram consideradas três categorias: empregado(a), desempregado(a) e reformado(a). Relativamente a esta variável foram verificadas diferenças estatisticamente significativas no QOL para os fatores Bem-estar Financeiro, $F(2,45)=7,56, p$ $=0,001,\left(\eta^{2}=0,25\right)$, e Educação, $F(2,45)=5,86$, $p=0,005,\left(\eta^{2}=0,21\right)$, e também no Resultado Total da SWLS, $F(2,45)=9,01, p=0,001\left(\eta^{2}\right.$ $=0,29)$. Na EPS, as diferenças se localizam $\left(\eta^{2}\right.$ $=0,14$ a 0,29$)$, nas subescalas Aconselhamento, $F(2,45)=6,67, p=0,003$; Aliança Fiável, $F(2,45)=6,86, p=0,003$; Reafirmação de Valor, $F(2,45)=3,68, p=0,033$; Oportunidade de Prestação de Cuidados, $F(2,45)=9,01, p=0,001$; Vinculação, $F(2,45)=4,56, p=0,016$; Apoio Íntimo, $F(2,45)=9,39, p<0,001$; Apoio Casual, $F(2,45)=3,94, p=0,027$; e Escala Total da EPS, $F(2,45)=8,62, p=0,001$. No que toca à qualidade de vida familiar (QOL), o teste de Bonferroni verificou que no fator Bem-Estar Financeiro os sujeitos DV empregados apresentam um resultado mais elevado $(M=18,83, D P=3,41)$ do que os sujeitos desempregados $(M=14,90, D P$ $=3,54)$ e reformados $(M=14,80, D P=3,76)$. No fator Educação, os sujeitos DV empregados obtiveram resultados mais elevados $(M=7,30, D P$ $=1,40)$ do que os reformados $(M=5,80, D P=$ 1,52). Ao nível da satisfação com a vida (SWLS), se registaram resultados mais elevados por parte dos sujeitos DV empregados $(M=18,48, D P=$ $3,44)$ em comparação com os desempregados $(M$ $=12,80, D P=3,85)$ e reformados $(M=15,20$, $D P=4,06)$. Relativamente ao apoio social per- cebido (EPS), foi verificado que os sujeitos DV empregados e desempregados alcançaram resultados superiores nas subescalas Aconselhamento, Aliança Fiável, Oportunidade de Prestação de Cuidados, Apoio Íntimo e Escala Total da EPS relativamente aos sujeitos DV reformados. Os sujeitos DV empregados apresentaram maiores pontuações nas subescalas Reafirmação de Valor e Apoio Casual $(M=12,78, D P=1,13$ e $M=$ $25,61, D P=2,11$, respetivamente) em comparação com sujeitos reformados $(M=11,73, D P=$ 1,10 e $M=23,80, D P=1,97$, respetivamente). Por fim, os sujeitos DV desempregados apresentaram resultados mais elevados na subescala Vinculação $(M=13,40, D P=2,12)$ do que os sujeitos reformados $(M=11,27, D P=1,67)$.

\section{Discussão}

O presente estudo pretendeu representar um contributo para a compreensão da percepção da qualidade de vida familiar, satisfação com a vida e apoio social percebido entre sujeitos com deficiência visual e sujeitos normovisuais. Assim, foi possível verificar que, comparando a caraterização das duas amostras ao nível das variáveis demográficas, apenas foi encontrada uma diferença estatisticamente significativa na variável situação profissional, com o grupo DV a englobar mais sujeitos aposentados. Estes resultados são congruentes com a relevância dada na literatura sobre a evidência de uma percentagem considerável de sujeitos DV aposentados (e.g., Bittencourt \& Hoehne, 2006; Cimarolli \& Wang, 2006; Guerette \& Smedema, 2011), já que estes, devido à sua incapacidade ao nível da percepção visual, acabam por se aposentar numa idade mais jovem do que a maioria da população geral na ausência de incapacidade comprovada.

Primeiramente importa destacar o estudo realizado acerca da consistência interna dos itens dos instrumentos de avaliação utilizados no presente estudo - QOL, SWLS e EPS - na amostra de sujeitos DV, dado que estes instrumentos não haviam sido validados para a população com deficiência visual, no sentido de verificar a adequabilidade da utilização dos mesmos com sujeitos DV. De acordo com Almeida e Freire (2008), o 
número de itens constituinte de um instrumento influencia o valor do coeficiente utilizado para o cálculo de fidelidade dos resultados, no sentido de um maior número de itens promover o seu aumento. Em todo o caso, os resultados obtidos nesta pesquisa para os três instrumentos apontaram para um valor de coeficiente alfa de Cronbach classificado como "muito bom" $\left(\alpha_{\mathrm{QOL}}=0,90\right.$; $\alpha_{\mathrm{SWLS}}=0,83 ; \alpha_{\text {EPS }}=0,92$; Almeida \& Freire, 2008), resultados estes favoráveis à utilização dos três instrumentos na amostra DV do presente estudo, ainda que o número de itens seja distinto em cada um dos três instrumentos (QOL $=40$; SWLS $=5 ; \mathrm{EPS}=24)$. Todavia, é de notar que os valores de coeficiente alfa de Cronbach obtidos no estudo de consistência interna dos itens dos vários fatores, subescalas e resultados totais dos três instrumentos utilizados, se mostraram inferiores no grupo DV relativamente aos valores do grupo de comparação. Estas diferenças podem dever-se não só a características próprias da amostra de pessoas com deficiência visual, como também a eventuais influências do recurso ao contacto telefónico para aplicação do protocolo em grande maioria dos sujeitos da amostra DV $(90,4 \%)$.

No que concerne ao estudo comparativo entre a amostra DV e a amostra normovisual relativamente à percepção da qualidade de vida familiar, se destaca a diferença estatisticamente significativa encontrada no fator Tempo, com um resultado mais elevado no grupo DV do que no grupo de comparação. Sobre este resultado não foi possível construir uma compreensão com base na literatura dada a ausência de estudos acerca da percepção da qualidade de vida familiar em sujeitos com deficiência visual, em contraste com as pesquisas sobre o impacto da qualidade de vida individual nesta população. Assim, atendendo aos itens englobados por este fator (e.g., "Quantidade de tempo livre", "Tempo para a família"), e dada a elevada percentagem de reformados na amostra DV, poderá ser considerado que estes possuem uma maior quantidade de tempo disponível e que esta disponibilidade se reflete em maior qualidade no aproveitamento do mesmo, comparativamente com o grupo de normovisuais que possui maior número de sujeitos empregados e, por isso, como menos tempo livre disponível. Pelo contrário, o resultado inferior dos sujeitos do grupo DV no fator Mass Media em relação ao grupo de comparação poderá estar relacionado com a sua impossibilidade em ter a mesma percepção sensorial dos programas de televisão ou dos jornais e revistas tal como têm os normovisuais, manifestando a desvantagem no acesso à informação (apesar de, hoje em dia, existir um avanço considerável dos meios tecnológicos e comunicacionais). Por outro lado, os resultados mais elevados do grupo DV no fator Emprego apontam para uma boa percepção de si próprios enquanto profissionais na execução de funções relacionadas com o seu emprego, e o reconhecimento dessas competências pelos outros poderá se considerar como uma vantagem, procurando explicar os resultados mais elevados obtidos comparativamente aos sujeitos do grupo de comparação.

No que se refere à percepção da satisfação com a vida, a literatura aponta no sentido de existirem diferenças estatisticamente significativas entre sujeitos com deficiência visual e sujeitos normovisuais, na medida em que os segundos se consideram como mais satisfeitos (e.g., Good et al., 2008). Apesar de não alcançar o limiar de significância estatística, os resultados do presente estudo vão no sentido da literatura dado que o grupo DV apresentou resultados ligeiramente inferiores aos do grupo de comparação.

Quanto à percepção do apoio social percebido, o grupo de comparação obteve resultados tendencialmente superiores ao grupo DV, tendo apenas sido verificada uma diferença estatisticamente significativa na subescala Vinculação (e.g., "Sinto que não tenho relações próximas com outras pessoas"), com o grupo de sujeitos normovisuais a registar um resultado mais elevado. É colocada a possibilidade de os sujeitos DV procurarem desde cedo a sua autonomia e independência como forma de combater o preconceito de que "quem não vê" precisa de alguém com visão normal para cuidar e executar tarefas (e.g., cozinhar, cuidar da casa/dos filhos, trabalhar), podendo haver lugar para que relações próximas de confiança e intimidade com figuras de suporte, promotoras de interdependência, afeto e re- 
conforto, acabem por se confundir com relações de dependência (Bifulco \& Thomas, 2013).

Por fim, foi realizada a análise do impacto de algumas variáveis demográficas mais relevantes na literatura com sujeitos DV (e.g., Bittencourt \& Hoehne, 2006; Cimarolli \& Wang, 2006; Guerette \& Smedema, 2011; Langelaan et al., 2007): sexo, idade e situação profissional nos resultados. Na presente pesquisa os homens obtiveram resultados superiores aos das mulheres ao nível da qualidade de vida familiar, congruente com a literatura existente que refere que sujeitos com deficiência visual do sexo masculino obtêm resultados mais elevados na percepção da qualidade de vida (e.g., Holbrook et al., 2009). Na percepção da qualidade dos Mass Media o resultado obtido entre homens e mulheres foi idêntico, indicando que tanto o sexo masculino, quanto o feminino tendem a considerar os Mass Media da mesma forma dada a sua condição de DV. A subescala Vinculação, no que se refere ao apoio social percebido, também obteve resultados idênticos entre ambos os sexos, permitindo formular a hipótese de que os homens e as mulheres desta amostra DV tiveram a mesma percepção acerca das suas relações de intimidade e partilha com entes próximos.

$\mathrm{Na}$ variável idade, a literatura indica que mais idade está associada a uma maior percepção da satisfação com a vida (e.g., Good et al., 2008; Guerette \& Smedema, 2011), no entanto, no presente estudo não foi encontrada uma diferença estatisticamente significativa nesse sentido. Por outro lado, no que respeita ao apoio social percebido, foram as faixas etárias mais jovens (18-35 anos) que obtiveram resultados mais elevados, o que poderá estar relacionado com o facto de sujeitos DV mais jovens terem acesso a um conjunto de meios e serviços que lhes permitem construir e manter uma rede de suporte mais alargada.

No que diz respeito à situação profissional, as diferenças estatisticamente significativas na percepção do bem-estar financeiro e da educação indicam que sujeitos DV em situação de empregabilidade obtiveram resultados mais elevados do que os sujeitos DV aposentados, no que respeita à qualidade de vida familiar. Dado que a empregabilidade desempenha um importante papel no combate ao isolamento social, contribuindo para uma maior autoestima e autonomia financeira (Cimarolli \& Wang, 2006; Crudden, 2012; Jorge, 2006), pode se considerar como mais favorável deste ponto de vista do que uma situação de aposentadoria. No presente estudo a diferença estatisticamente significativa obtida na percepção da satisfação com a vida por parte de sujeitos DV indicou resultados mais elevados por parte dos sujeitos empregados relativamente aos sujeitos desempregados e aposentados, o que vai ao encontro da literatura (e.g., Cimarolli \& Wang, 2006; Guerette \& Smedema, 2011; Mcllvane \& Reinhardt, 2001), uma vez que a empregabilidade se constitui como um fator importante, influente e benéfico para o bem-estar dos indivíduos. Em relação ao apoio social percebido foi verificado que sujeitos DV reformados apresentaram resultados inferiores ao nível da escala total, bem como nas respetivas subescalas. Comparativamente com sujeitos desempregados que, por procurarem emprego, possivelmente têm maior oportunidade para contactar com outras pessoas, os sujeitos reformados poderão não mostrar tendência a alargar a sua rede de contactos interpessoais, levando-os a um "maior fecho" da rede de suporte e, consequentemente, um menor nível de percepção de apoio social recebido.

\section{Conclusão}

A presente pesquisa procurou investigar a percepção da qualidade de vida familiar, satisfação com a vida e apoio social percebido de sujeitos com deficiência visual e de sujeitos normovisuais, contribuindo para uma reflexão sobre a influência e o impacto da deficiência visual enquanto condição crónica, principalmente no que respeita à percepção da qualidade de vida familiar pelo indivíduo. $\mathrm{O}$ número de sujeitos na amostra de pessoas com DV $(N=52)$, nem sempre fácil de obter, consta também como uma mais-valia. Ainda assim, é relevante assinalar que o procedimento de amostragem não probabilística, bem como a não representatividade da amostra, apesar do número total de sujeitos, não 
permite elaborar conclusões sobre a adequabilidade dos instrumentos utilizados à população portuguesa com deficiência visual e, como tal, as generalizações dos resultados obtidos devem ser efetuadas com precaução - no entanto, o presente estudo representa uma primeira validação dos três instrumentos com sujeitos com deficiência visual. Outras limitações se depararam com a reduzida quantidade de literatura disponível sobre o impacto da deficiência visual no sujeito, principalmente no que respeita à qualidade de vida familiar, se colocando como uma barreira na interpretação dos resultados obtidos. Por fim, e apesar de na literatura o tipo de deficiência visual - baixa visão e cegueira - ser uma variável com impacto ao nível da qualidade de vida (e.g., Bittencourt \& Hoehne, 2006), no caso do presente estudo não foi possível averiguar tal condição devido ao reduzido número de sujeitos com baixa visão relativamente ao número de sujeitos com cegueira.

Em futuras investigações, é sugerida a replicação do estudo aqui apresentado recorrendo a amostras de maiores dimensões. Não obstante, se recomenda que mais estudos sejam realizados recorrendo à população com deficiência visual, nomeadamente, o estudo das diferenças na percepção da qualidade vida familiar, satisfação com a vida e apoio social percebido em sujeitos DV que tenham, ou não, usufruído de um processo de reabilitação, ou entre as duas dimensões da deficiência visual - a baixa visão e a cegueira -, procurando, deste modo, alargar o leque de entendimento sobre o impacto de características específicas da deficiência visual na vida pessoal e familiar de indivíduos. É, ainda, sugerido aprofundamento de estudos relacionados com o desenvolvimento de vinculação entre sujeitos com deficiência visual e os seus familiares, com vista a melhor compreender a importância do papel desempenhado por estes últimos face à presença de doença crónica (Rolland, 1994). Por último, é proposta a possibilidade de incluir instrumentos de medida que permitam avaliar a percepção da qualidade de vida familiar, satisfação com a vida e apoio social percebido nos programas de intervenção direcionados para indivíduos com deficiência visual e suas famílias, antes e depois do processo interventivo, no sentido de averiguar a existência de diferenças. Na sua essência, pretender-se-ia que estes programas de intervenção promovessem e aperfeiçoassem as competências de pessoas com deficiência visual em várias componentes (e.g., pessoal, familiar, social, profissional), com especial enfoque nos aspetos que pudessem constituir maior dificuldade por parte destes sujeitos.

Deste modo, se considera que os objetivos inicialmente propostos foram respondidos, permitindo a reflexão sobre a importância de continuar em busca de um conhecimento mais aprofundado acerca do impacto da deficiência visual sobre a qualidade de vida familiar, satisfação com a mesma e o apoio social percebido nos sujeitos, capaz de constituir mais um passo para a implementação de uma intervenção precoce e direcionada para as necessidades emocionais e instrumentais dos sujeitos com deficiência visual e respetiva rede de apoio.

\section{Referências}

Almeida, L. S., \& Freire, T. (2008). Metodologia da investigação em Psicologia e Educação (5. ed.). Braga, Portugal: Psiquilíbrios.

Bambara, J. K., Wadley, V., Owsley, C., Martin, R. C., Porter, C., \& Dreer, L. E. (2009). Family functioning and low vision: A systematic review. Journal of Visual Impairment \& Blindness, 103(3), 137-149. Retrieved from: http:// search.proquest.com/docview/222022324?acco untid $=43959$

Bifulco, A., \& Thomas, G. (2013). Understanding adult attachment in family relationships: Research, assessment, and intervention. Abingdon, UK: Routledge.

Bittencourt, Z., \& Hoehne, L. (2006). Qualidade de vida de deficientes visuais. Medicina (Ribeirão Preto), 39(2), 260-264.

Cimarolli, V. R., \& Wang, S. (2006). Differences in social support among employed and unemployed adults who are visually impaired. Journal of Visual Impairment \& Blindness, 100(9), 545-556. Retrieved from http://search.proquest. com/docview/222056111 ?accountid $=43959$

Crudden, A. (2012). Transition to employment for students with visual impairments: Components 
for success. Journal of Visual Impairment \& Blindness, 106(7), 389-399. Retrieved from http://search.proquest.com/docview/103042365 4? accountid $=43959$

Fagulha, T., Duarte, M. E., \& Miranda, M. J. (2000). A "qualidade de vida": Uma nova dimensão psicológica? Psychologica, 25, 5-17.

Good, G. A., La Grow, S., \& Alpass, F. (2008). An age-cohort study of older adults with and without visual impairments: Activity, independence, and life satisfaction. Journal of Visual Impairment \& Blindness, 102(9), 517-527. Retrieved from http://search.proquest.com/docview/22202 2999? accountid $=4395$

Guerette, A. R., \& Smedema, S. M. (2011). The relationship of perceived social support with wellbeing in adults with impairments. Journal of $\mathrm{Vi}$ sual Impairment \& Blindness, 105(7), 425-439. Retrieved from http://search.proquest.com/docv iew $/ 880321042$ ? accountid $=43959$

Holbrook, E. A., Caputo, J. L., Perry, T. L., Fuller, D. K., \& Morgan, D. W. (2009). Physical activity, body composition, and perceived quality of life of adults with visual impairments. Journal of Visual Impairment \& Blindness, 103(1), 17-29. Retrieved from http://search.proquest.com/docv iew/222065042? accountid=43959

Hu, X., Summers, J. A., Turnbull, A., \& Zuna, N. (2011). The quantitative measurement of family quality of life: A review of available instruments. Journal of Intellectual Disability Research, 55(12), 1098-1114. doi:10.1111/ j.1365-2788.2011.01463.x

Jorge, S. C. (2006). A inserção do deficiente visual no mercado de trabalho: Um estudo empírico no concelho de Coimbra (Dissertação de mestrado não publicada, Faculdade de Psicologia e de Ciências da Educação, Universidade de Coimbra, Portugal).

La Grow, S., Alpass, F., Stephens, C., \& Towers, A. (2011). Factors affecting perceived quality of life of older persons with self-reported visual disability. Quality of Life Research, 20(3), $407-$ 413. doi:10.1007/s11136-010-9758-6

Langelaan, M., De Boer, M. R., Van Nispen, R. M. A., Wouters, B., Moll, A. C., \& Rens, H. M. B. (2007). Impact of visual impairment on quality of life: A comparison with quality of life in the general population and with other chronic conditions. Ophthalmic Epidemiology, 14, 119-126. doi:10.1080/09286580601139212
McIlvane, J., \& Reinhardt, J. (2001). Interactive effect of suport from family and friends in visually impaired elders. The Journals of Gerontology, 56(6), 374-382. doi:10.1093/geronb/56.6.P374

Moreira, J. M., \& Canaipa, R. (2007). A Escala de Provisões Sociais: Desenvolvimento e validação da versão portuguesa da "Social Provisions Scale". Revista Ibero-Americana de Diagnostico y Evaluacion Psicologica, 24(2), 23-58.

Pallant, J. (2005). SPSS survival manual. A step by step guide to data analysis using SPSS for Windows (Version 12) (2 $2^{\text {nd }}$ ed.). Sidney, Australia: Allen \& Unwin.

Rolland, J. S. (1994). Families, illness, and disability. An integrative treatment model. New York: Basic Books.

Silveira, M. J., \& Sequeira, A. (2002). A saúde mental na inserção social da pessoa com cegueira adquirida. Análise Psicológica, 3(20), 449-470. Recuperado em http://repositorio.ispa.pt/handle/10400.12/1852

Simões, A. (1992). Ulterior validação de uma escala de satisfação com a vida (SWLS). Revista Portuguesa de Pedagogia, 26(3), 503-515.

Simões, J. M. L. (2008). Qualidade de vida: Estudo de validação para a população portuguesa (Dissertação de mestrado não publicada, Faculdade de Psicologia e de Ciências da Educação, Universidade de Coimbra, Portugal).

Stelmack, J. (2001). Quality of life of low-vision patients and outcomes of low-vision rehabilitation. Optometry \& Vision Science, 78(5), 335-342.

World Health Organization. (2004). ICD-10: International statistical classification of diseases and related health problems - $10^{\text {th }}$ Revision. Geneve, Switzerland: W. H. O.

World Health Organization. (2012). 10 facts about blindness and visual impairment. Retrieved from http://www.who.int/features/factfiles/ blindness/en/index.html\#

World Health Organization. (2013). Prevention of blindness and visual impairment. Retrieved from http://www.who.int/blindness/causes/priority/en/index $5 . h t m l$ 